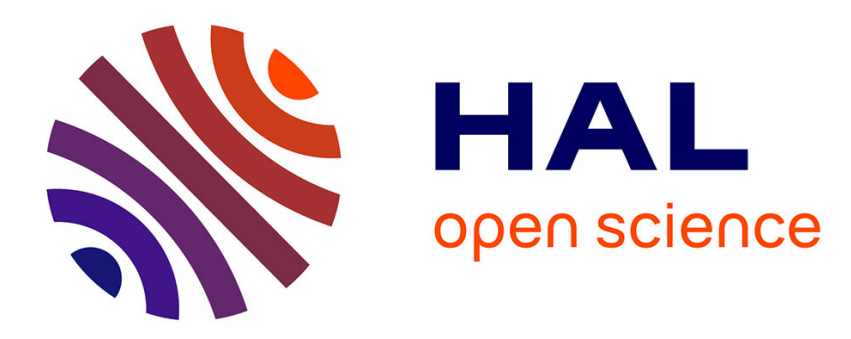

\title{
Simulation of the mechanical behaviour of woven fabrics at the scale of fibers
}

Damien Durville

\section{To cite this version:}

Damien Durville. Simulation of the mechanical behaviour of woven fabrics at the scale of fibers. International Journal of Material Forming, 2010, 3, pp.1241-1251. 10.1007/s12289-009-0674-7 . hal00495110

\section{HAL Id: hal-00495110 https://hal.science/hal-00495110}

Submitted on 25 Jun 2010

HAL is a multi-disciplinary open access archive for the deposit and dissemination of scientific research documents, whether they are published or not. The documents may come from teaching and research institutions in France or abroad, or from public or private research centers.
L'archive ouverte pluridisciplinaire HAL, est destinée au dépôt et à la diffusion de documents scientifiques de niveau recherche, publiés ou non, émanant des établissements d'enseignement et de recherche français ou étrangers, des laboratoires publics ou privés. 


\title{
Simulation of the mechanical behaviour of woven fabrics at the scale of fibers
}

\section{Damien Durville}

Received: date / Accepted: date

\begin{abstract}
A general approach to the mechanical behaviour of woven fabrics at the scale of individual fibers is proposed in this paper. In order to simulate the behaviour of samples of woven fabrics, all fibers constituting these samples are taken into account in the model, and particular attention is focused on the detection and modeling of contact-friction interactions occuring within the collection of fibers. The global problem is set within a large deformation framework, and is solved using an implicit algorithm. The developed methods are first employed to compute the unkown initial configuration of woven structures by simulating the weaving process. Various loading cases can then be applied in order to identify the mechanical properties of such materials. Numerical results about samples made of nearly 500 fibers are given to show the ability of the method to handle representative examples. Very useful informations at the scale of individual fibers are obtained from these simulations and should help to understand the mechanisms at microscopic scale that rule the complex nonlinear behaviour of woven fabrics.
\end{abstract}

Keywords Woven fabrics · Mechanical properties · Textile materials · Contact-friction interactions · Finite element simulation

\section{Introduction}

As textile fibers are more used to manufacture technical fabrics or to reinforce composite materials, the need of mechanical characterization of textile materials is growing. If the global mechanical behaviour of fabrics can be investigated and identified through experiments, the simulation becomes a new means to explore the complex behaviour of such structures.

A woven fabric is constituted by components of two levels. At a first level, a fabric is made of interlacing tows, whereas at a second level tows are constituted by bundles

D. Durville

Laboratoire de Mécanique des Sols Structures et Matériaux (LMSSM)

CNRS UMR8579 - Ecole Centrale Paris

Grande Voie des Vignes - 92290 Chatenay-Malabry (France)

Tel.: +33-141131290 E-mail: damien.durville@ecp.fr 
of fibers. The modeling of woven fabrics can be tackled at three different scales. At a macroscopic scale, homogeneized models can be derived to represent the fabric as a plate or a shell. By developing models for yarns or tows, considered as continuous media, it is possible to build intermediate approaches to the behaviour of fabrics at a mesoscopic scale, considering the fabric as an assembly of interlacing tows. However, going down one more level, the mechanical behaviour of individual fibers has to be taken into account to describe the behaviour of woven structures at a microscopic scale.

Many approches are available in the literature concerning the modeling of fabrics at macroscopic and at mesoscopic scale [1, 5, 3, 4, 2]. One of the limits of these approaches is that they have to assume and to identify intermediate models to describe the behaviour of the threads, or the interactions between them.

To avoid to have to make assumptions about the behaviour of intermediate components, the approach presented here focuses on the microscopic scale, in order to understand and to predict mechanisms taking place at the scale of the fibers. This approach considers small samples of woven fabrics as collections of individual fibers undergoing large deformations, and needs to model not only the behaviour of all individual fibers, but also contact-friction interactions developed between them. The only characteristics required for the modeling are those of fibers, that are readily available. This approach, by describing the mechanical state of all individual fibers involved in the sample, is also useful for the prediction of damage, in particular due to fiber breakage.

The determination of the initial configuration of the woven fabric, which is unknown a priori, is a critical issue for for simulation at microscopic scale, since the initial geometry of each fiber has to be described. To obtain this initial configuration, it is necessary to simulate the weaving process. This preliminary task provides as results many useful informations about the arrangement of fibers in the fabric.

When dealing with woven fabrics at the scale of fibers, the difficulties lie in the detection and modeling of numerous contact-friction interactions taking place within the collection of fibers. Within the framework of large deformations, this leads to nonlinear problems that require optimized algorithms to be solved efficiently, especially when high numbers of degrees of freedom and contacts are considered. The recent improvements in computing capacities make the finite simulation at the scale of fibers a possible way to explore the complex behaviour of textile fabrics.

Similar approaches at microscopic scale can be found in literature. At the scale of fibers, for the computation of the initial configuration of braided structures or 3D interlock woven fabric, digital elements have been used by Miao 91. Since these digital elements have neither bending nor torsional stiffness, fibers must be tightened to find a solution. Finckh [8] proposed to simulate the weaving process and to apply dynamic loading cases using an explicit solver.

The present work 6, 6] is based on the use of an implicit solver, in accordance with the quasi-static framework in which the problem is set. Compared to an explicit solvers, an implicit offers a good control of the quality of the solution, but needs efficient numerical algorithms to solve large loading increments and save computation time.

To introduce the global approach, a first section is dedicated to the beam model employed to represent fibers. The contact modeling, including geometrical and mechanical aspects, is then described. The application of boundary conditions to the ends of tows and fibers, using rigid bodies and average binding conditions are presented in the next section. The simulation of the weaving process is explained and results on two samples with different weave architectures are shown. The initial configuration of these 


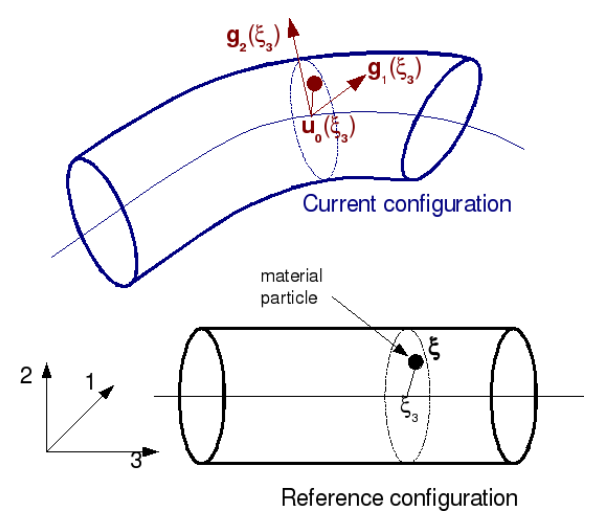

Fig. 1 The three kinematical vectors used to define the placement in the kinematical beam model

samples is first computed by simulating the weaving process, and biaxial and shear tests are then simulated to identify their mechanical properties.

\section{$23 \mathrm{D}$ finite strain beam model}

\subsection{Introduction}

Beam models are usually based on the assumption that beam cross-sections remain rigid. The motion of cross-sections is described by two vector fields (six degrees of freedom) : one translation for the center of the section, and one rotation. These models present two main disadvantages : the handling of large rotations requires complex formulations, and they do not take any account of possible deformations of crosssections. To overcome these limits, an enriched kinematical model, based on three vector fields and nine degrees of freedom was developed.

\subsection{Enriched kinematical beam model}

The enriched kinematical beam model is based on a first order Taylor expansion of the placement of any material particle of the beam with respect to the line of centroids. Denoting $\boldsymbol{\xi}\left(\xi_{1}, \xi_{2}, \xi_{3}\right)$ a material particle defined in the reference configuration (see Fig. 11), a first order expansion of its placement $\mathbf{x}(\boldsymbol{\xi})$ can be expressed as follows :

$$
\mathbf{x}(\boldsymbol{\xi})=\mathbf{x}\left(0,0, \xi_{3}\right)+\xi_{1} \frac{\partial \mathbf{x}}{\partial \xi_{1}}\left(0,0, \xi_{3}\right)+\xi_{2} \frac{\partial \mathbf{x}}{\partial \xi_{2}}\left(0,0, \xi_{3}\right)+o\left(\xi_{1}, \xi_{2}\right)
$$

Denoting respectively $\mathbf{x}_{0}, \mathbf{g}_{1}$ and $\mathbf{g}_{2}$ the three vectors fields introduced in the above expansion, which depend only on the curvilinear abscissa $\xi_{3}$ of the particle, a kinematical model can be based on the following expression of the placement of any particle :

$$
\mathbf{x}(\boldsymbol{\xi})=\mathbf{x}_{0}\left(\xi_{3}\right)+\xi_{1} \mathbf{g}_{1}\left(\xi_{3}\right)+\xi_{2} \mathbf{g}_{2}\left(\xi_{3}\right) .
$$

In this model, three vectors are used to describe any cross-section of the beam : the vector $\mathbf{x}_{0}$ which defines the position of its center, and two other vectors, $\mathbf{g}_{1}$ and 
$\mathbf{g}_{2}$, called section vectors, which determine the orientation of the section. According to this expression for the placement, the displacement $\mathbf{u}(\boldsymbol{\xi})$ of any particle is derived as follows :

$$
\mathbf{u}(\boldsymbol{\xi})=\mathbf{u}_{0}\left(\xi_{3}\right)+\xi_{1} \mathbf{h}_{1}\left(\xi_{3}\right)+\xi_{2} \mathbf{h}_{2}\left(\xi_{3}\right) .
$$

With this kinematical model based on three vector fields, beam cross-sections are assumed to remain plane. However, since the norm of section vectors and the angle they form can vary, planar deformations of cross-sections can be considered.

\subsection{Strains and constitutive law for the beam model}

With this model, because of the consideration of planar deformations of cross-sections, no term of the Green-Lagrange strain tensor is constrained to be zero, and fully threedimensional effects, such as Poisson's effect, are taken into account. The fact that the strain tensor is fully three-dimensional allows to use usual three-dimensional constitutive laws, taking into account strains in all directions.

\section{Contact and friction modeling}

\subsection{Introduction}

The high number of contacts involved in the considered collections of fibers makes the detection and modeling of contact-friction interactions a crucial issue of the problem. The taking into account of contact-friction interactions requires first to detect contacts which are occuring within the collection of beams and which are continuously evolving due to the large relative displacements allowed between fibers. Once contacts have been detected, interactions forces developed in normal and tangential directions need to be introduced by means of appropriate contact and friction models, allowing the use of efficient algorithms.

\subsection{Geometrical detection of contact}

Because of the continuous evolution of contact during the loading, the contact detection has to be repeated during the solving of the problem, and the process to detect contact needs therefore to be efficient in order not to consume too much computational time.

Contact configurations between fibers can be very different depending on their relative positions. Very localized and reduced contact areas can be observed at crossings between fibers of two different tows, whereas continuous contact lines may appear between two neighboring fibers of the same tow.

Methods to detect contact between 3D beams can be found in literature [10, 11]. They often lie on the search of nearest distances between beams, which may consume much computation time when a high number of fibers is considered. In order to consider the various possible configurations between fibers, even with a high number of fibers, we propose a method based on the determination, in regions where contact is likely to occur, of discrete contact elements, constituted by pairs of material particles predicted to enter into contact (see Fig. 2). A contact element, denoted $E_{c}^{i j}\left(\mathbf{x}_{G}\right)$, is defined by 


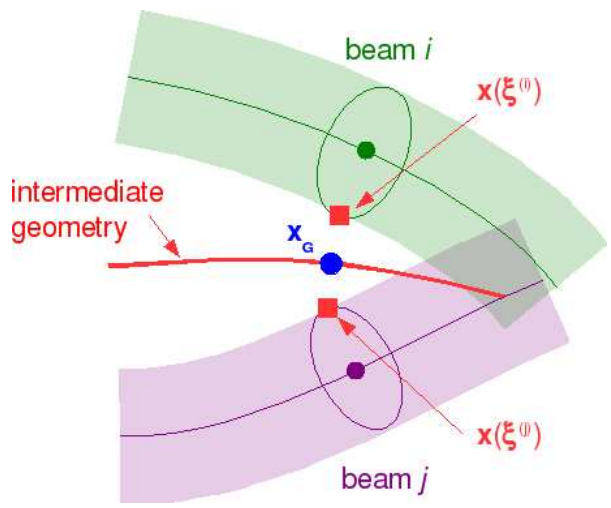

Fig. 2 Definition of a contact element

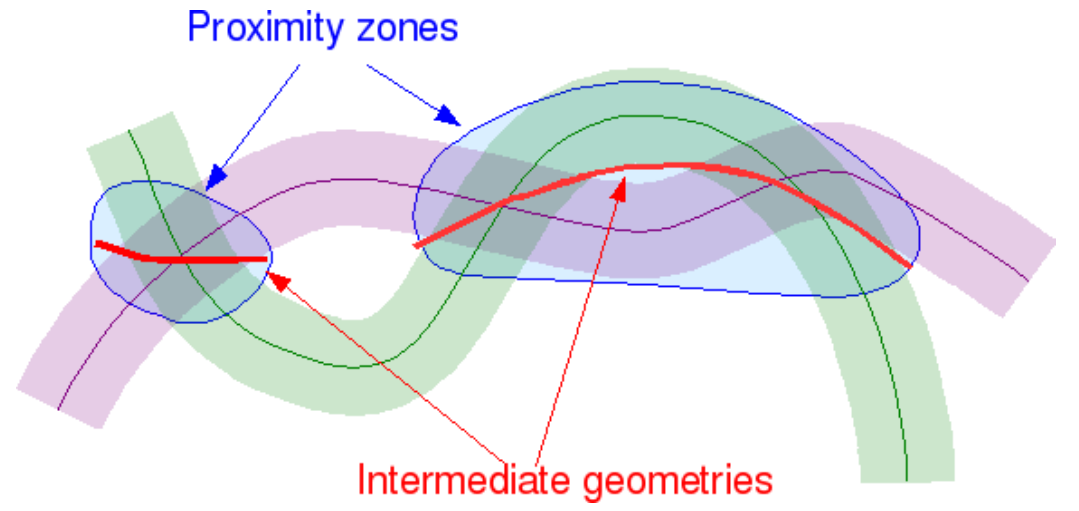

Fig. 3 Definition of intermediate geometries based on proximity zones

the pair of material particles, $\boldsymbol{\xi}^{(i)}$ and $\boldsymbol{\xi}^{(j)}$, belonging to two different beams $i$ and $j$, that are predicted to enter into contact at $\mathbf{x}_{G}$ :

$$
E_{c}^{i j}\left(\mathbf{x}_{G}\right)=\left(\boldsymbol{\xi}^{(i)}, \boldsymbol{\xi}^{(j)}\right) \text { such that } \mathbf{x}\left(\boldsymbol{\xi}^{(i)}\right)=\mathbf{x}\left(\boldsymbol{\xi}^{(j)}\right)=\mathbf{x}_{G} .
$$

The process of determination of contact elements follows three successive stages to determine these contact elements. First, regions where contact is likely to occur, and which are called proximity zones, are looked for. A proximity zone is defined as two parts of beam centroidal lines which are stated to be close enough (Fig. 3). These proximity zones are obtained for each pair of beams in the collection, using a coarse discretization on one of the beam, and searching for the lowest distance to the other beam.

Once zones of proximity have been determined, an intermediate geometry is defined for each zone, as the average between the two parts of lines close to each other (Fig. 3. The purpose of this intermediate geometry is to approximate the geometry of the contact area. Contact elements are the defined at discrete points regularly distributed along this intermediate geometry, by looking for the two material particles on the surface of beams which are likely to enter into contact at these discrete positions. To do this, a plane orthogonal to the intermediate geometry is used to determine two 


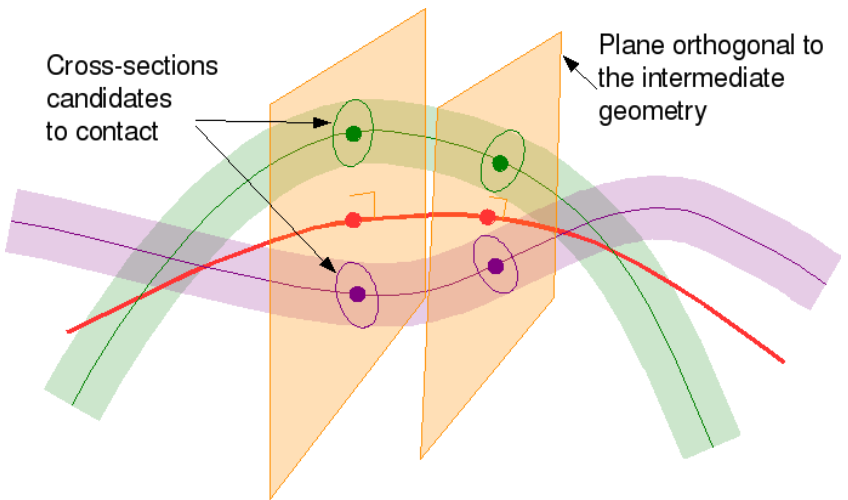

Fig. 4 Determination of couples of cross-sections candidates to contact

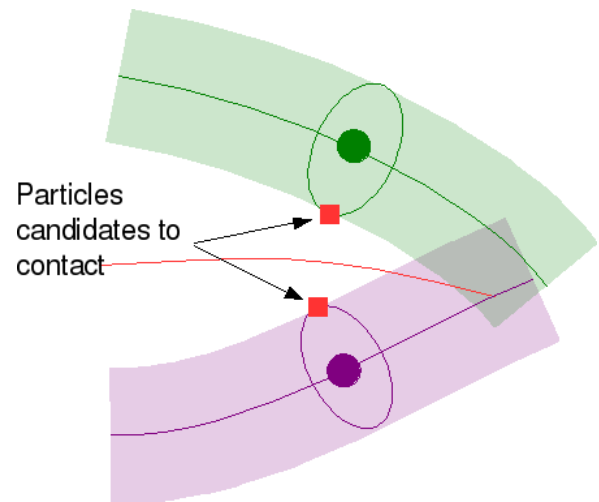

Fig. 5 Selection of material particles on the borders of cross-sections to create a contact element

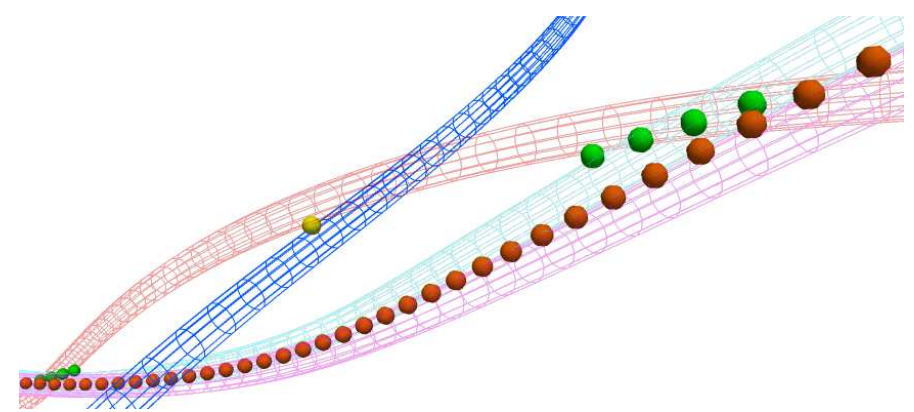

Fig. 6 Example of determination of contact elements (colored spheres) between fibers in different configurations

cross-sections whose centers are located at the interesection between these planes and the centroidal lines (Fig. (4). Particles candidate to contact are then accurately located on the edge of the cross-sections using geometrical criteria (Fig. E). 


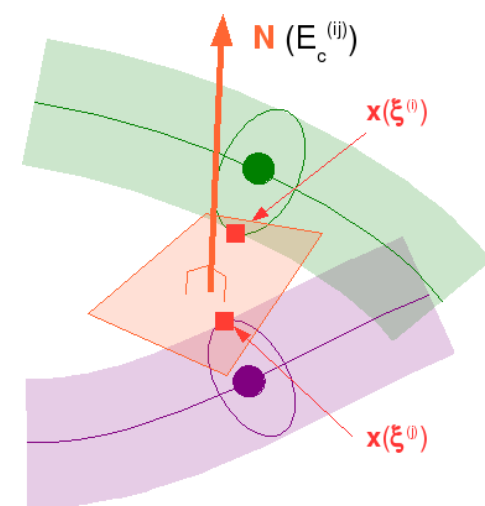

Fig. 7 Definition of a normal direction for a contact element

\subsection{Formulation of contact conditions}

In order to formulate linearized contact conditions, a normal direction, denoted $\mathbf{N}\left(E_{c}^{i j}\right)$, is associated to each contact element $E_{c}^{i j}$. This direction sets the direction according to which distance between interacting particles will be measured (Fig. 7). This contact direction is generally taken as the one between the two centers of cross-sections involved in contact.

In order to prevent interpenetration between beams, kinematical conditions are set for all contact elements. These conditions prescribe that the distance between particles of a contact element, measured according to the normal contact direction, must remain positive. This distance, defined as the gap, is expressed for each contact element as follows :

$$
\operatorname{gap}\left(E_{c}^{i j}\left(\mathbf{x}_{G}\right)\right)=\left(\mathbf{x}\left(\boldsymbol{\xi}^{(i)}\right)-\mathbf{x}\left(\boldsymbol{\xi}^{(j)}\right), \mathbf{N}\left(E_{c}^{i j}\right)\right) \geq 0
$$

\subsubsection{Constitutive laws for contact and friction}

A constitutive law for normal contact is considered in order to link normal reactions to the gaps measured at contact elements. This constitutive law has the form of a penalty law, and is regularized by a quadratic part for very small penetrations in order to stabilize the contact algorithm (see Fig. 8). The penalty coefficient is adjusted for each proximity zone in order to limit the maximum penetration for each zone. As far as the friction is concerned, we use a Coulomb's law including a small reversible elastic displacement before sliding occurs.

\subsubsection{Global algorithm to solve the nonlinear problem}

To solve the problem for each loading increment, imbricated loops are introduced to iterate on the different nonlinearities. For each increment, with a first level loop, iterations are made on the process of contact determination to generate contact elements. Then, these contact elements being fixed, iterations are made at a second level on the normal contact directions. The third level loop is dedicated to iterations of a NewtonRaphson algorithm on all other nonlinearities (contact status, friction direction, finite strains). Because of these three embedded loops, the total number of iterations may be 

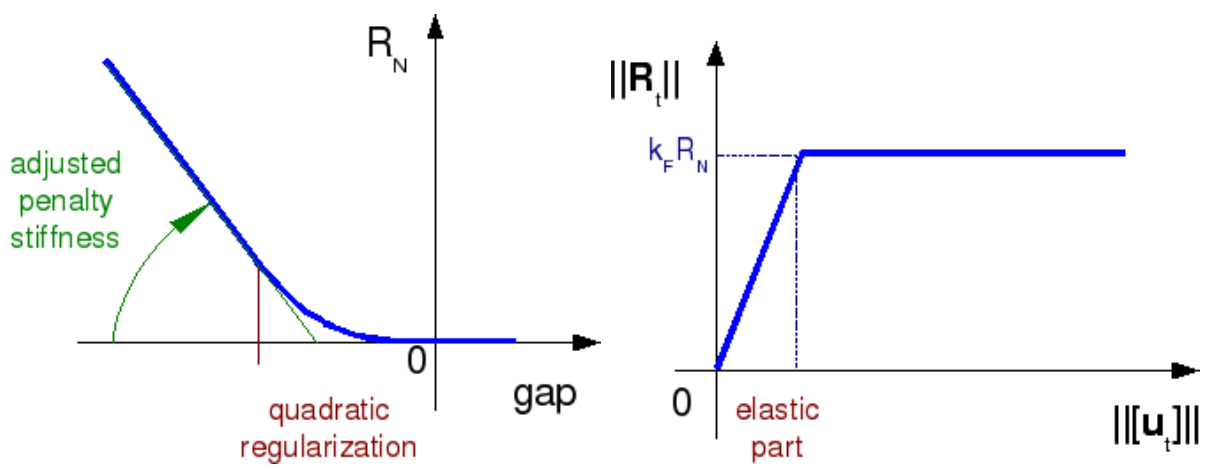

Fig. 8 Models for normal and tangential behaviour

high if a good rate of convergence is not obtained for the Newton-Raphson algorithm. Models characteristics need to be carefully adjusted for this purpose.

\section{Driving of boundary conditions}

\subsection{Introduction of rigid bodies}

As the considered samples of woven fabrics are made of components of different levels (tows and fibers), the application of boundary conditions to the edges of the sample is a delicate issue. The difficulty is that we want to apply globally loadings to each component (edge or end of tow), while allowing the subcomponents (tows for the edges, and fibers for tows) the possibility to move and rearrange, in order not to introduce artificial stiffnesses at the boundaries. In fact, if ends of tows and fibers were fixed respectively to the edges an the tows, this would rigidify the boundaries of the sample and would modify the global solution of the problem.

The goal of our boundary conditions is to distribute loads applied globally to the edges, to the subcomponents attached to these edges (respectively tows attached to edges, and fibers attached to tows) while allowing these subcomponents the possibility to move and rearrange.

To meet this objective, rigid bodies are introduced and attached to each edge and to each end of tow. These rigid bodies are used to apply proper boundary conditions to each edge and each end of tow. Boundary conditions to be applied to subcomponents are then defined with respect to these rigid bodies. Standard conditions can be used to prescribe a displacement or a force to each individual subcomponent. However, in order to allow subcomponents to move and rearrange, new conditions are set in average for all subcomponents attached to the same component. This way, it is possible to prescribe that all ends of tows follow in average the motion of a edge, or that the averaged motion of all ends of fibers corresponds to that of the tow. This combination of rigid bodies for components at different levels, and of averaged binding conditions provides a versatile way of driving boundary conditions, enabling to apply globally forces or displacements to the components of different levels, while allowing them the possibility to rearrange. 
4.2 Formulation of averaged binding conditions to the rigid bodies

Averaged conditions are aimed at binding globally the average displacements of a set of subcomponents to those of the moving rigid body they are attached to. As the motion of a rigid body is defined by six degrees of freedom (three translations and three rotations), six averaged binding conditions have to be set in order to control the average displacement and the average rotation of subcomponents. Let the rigid body be defined by the position of its center, $\mathbf{R}^{n}$ and its three moving directions $\left(\mathbf{D}_{i}^{n}\right)$ at step $n$, and the set of $N$ subcomponents be defined by the positions of corresponding nodes $\left(\mathbf{x}_{1}^{n}, \ldots, \mathbf{x}_{N}^{n}\right)$.

The condition on the average displacement means that the displacement of the barycenter of nodes of subcomponents to bind to the rigid body must be the same as the displacement of the rigid body, with respect to the moving frame attached to this rigid body, and is expressed as follows :

$$
\frac{1}{N}\left(\sum_{\lambda=1}^{N} \mathbf{x}_{\lambda}^{n}-\mathbf{R}^{n}, \mathbf{D}_{i}^{n}\right)=\frac{1}{N}\left(\sum_{\lambda=1}^{N} \mathbf{x}_{\lambda}^{n-1}-\mathbf{R}^{n-1}, \mathbf{D}_{i}^{n-1}\right), \forall i \in(1,2,3)
$$

In order to prevent the rotation of the set of subcomponents around the direction $\mathbf{D}_{i}$ of the rigid body, a condition is prescribed for the average of orthoradial displacements defined with respect to this direction. A radial direction $d_{r a d, \lambda}^{n-1}$ and an orthoradial direction $d_{\text {ortho, }}^{n-1}$ are defined in the previous configuration for each subcomponent in the following way :

$$
\begin{aligned}
& d_{\text {rad, } \lambda}^{n-1}=\frac{\mathbf{x}_{\lambda}^{n-1}-\mathbf{R}^{n-1}}{\left\|\mathbf{x}_{\lambda}^{n-1}-\mathbf{R}^{n-1}\right\|} \\
& d_{\text {ortho }, \lambda}^{n-1}=\frac{\mathbf{D}_{i}^{n-1} \times d_{\text {rad, }, \lambda}^{n-1}}{\left\|\mathbf{D}_{i}^{n-1} \times d_{\text {rad }, \lambda}^{n-1}\right\|}
\end{aligned}
$$

The orthoradial direction $d_{\text {ortho, } \lambda}^{n}$ in the current configuration is then obtained by a coordinate system transformation :

$$
d_{\text {ortho, }, \lambda}^{n}=\left(d_{\text {ortho, }, \lambda}^{n-1}, \mathbf{D}_{i}^{n-1}\right) \mathbf{D}_{i}^{n}
$$

The global condition on the average of orthoradial displacement around direction $\mathbf{D}_{i}$ is then expressed as follows :

$$
\frac{1}{N} \sum_{\lambda=1}^{N}\left(\mathbf{x}_{\lambda}^{n}-\mathbf{x}_{\lambda}^{n-1}, d_{\text {ortho, }, \lambda}^{n}\right)=0
$$

Equations and are of the same kind. They link together degrees of freedom of subcomponents and of the master rigid body by the means of a quadratic condition involving products of displacement field component. Thanks to these conditions, displacements of bond subcomponents are globally controled whereas each component remains free to move individually with respect to the whole set. 


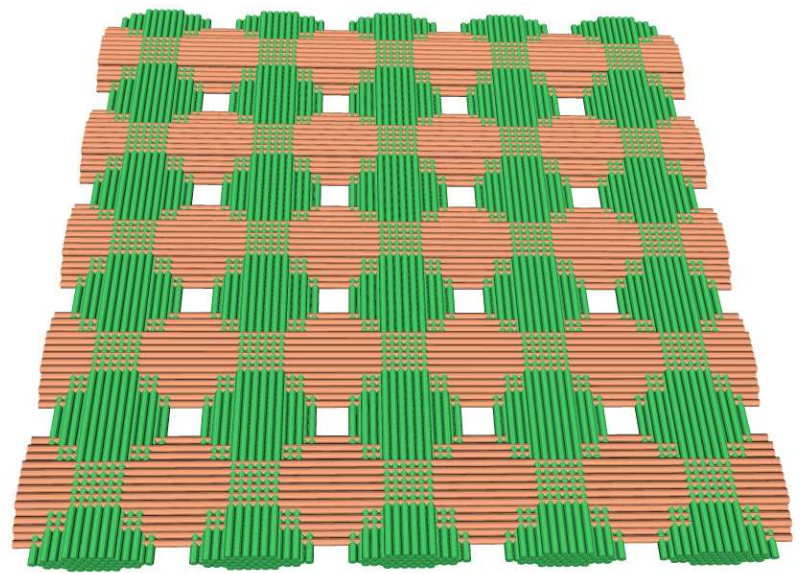

Fig. 9 Initial configuration of tows before weaving

\section{Computation of the initial configuration}

\subsection{Determination the initial configuration}

The trajectories of individual fibers in a piece of fabric can not be known a priori, but need to be computed by simulating in some way the weaving process. This task is performed starting from an arbitrary configuration where all tows are laying on the same plane, and are assumed to be straight and to have elliptical cross-sections. In this configuration (Fig. 9) tows are interpenetrating each other at crossings. According to the chosen weave pattern, it is possible to determine which tow must be below or above the other at each crossing. The weaving process is simulated by making fibers from different tows fulfil progressively the superimposing order defined by the weave pattern. For this purpose, during steps dedicated to the simulation of the weaving, the normal contact direction for contacts between fibers of different tows is oriented according to the order prescribed by the weave pattern at a given crossing. By this way, fibers from different tows are step-by-step separated until there is no more interpenetration between tows. Usual normal contact directions are then applied for all contacts to carry out a relaxation step in order to obtain the equilibrium configuration of the initial woven structure.

\subsection{Computation of the initial configuration for a plain weave and a twill weave}

To illustrate the simulation of the weaving process, two examples are presented here. The two examples use the same initial arrangement of ten tows, but two different weave architectures are considered to compute the initial configuration of the woven fabrics. The main data concerning these examples are gathered in Tab. 1. Each tow is made of 48 fibers, and the whole sample consists of 480 fibers.

The configurations obtained after the simulation of the weaving process are shown in Fig. 10 and 11 for the plain weave and the twill weave respectively. Interesting details can be viewed on Fig. 12 and 13 which represent slices of the samples at successive 


\begin{tabular}{lr}
\hline No. of warp tows & 5 \\
No. of weft tows & 5 \\
No. of fibers per tow & 48 \\
Total no. of fibers & 480 \\
No. of nodes & 38880 \\
No. of dofs & $\approx 350000$ \\
No. of contact elements & $\approx 80.000$ \\
\hline
\end{tabular}

Table 1 Characteristic figures of the simulations

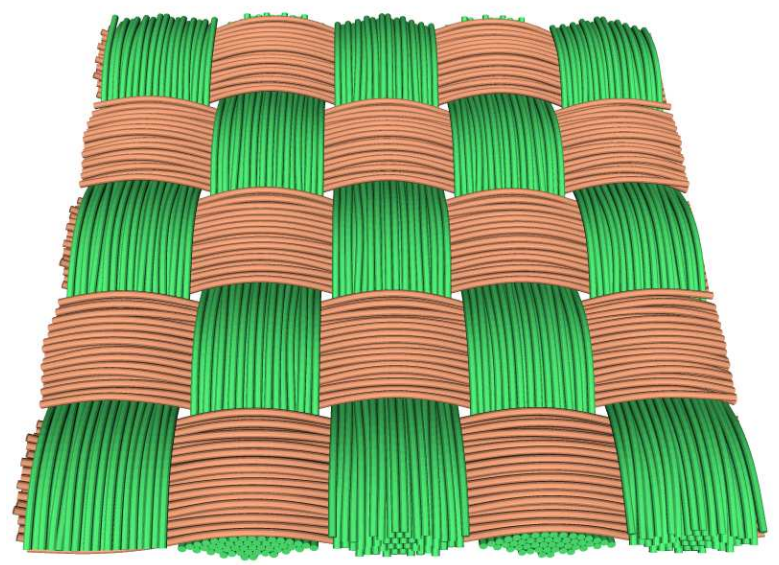

Fig. 10 Configuration after weaving for the plain weave sample

steps during the weaving process. The evolution of fibers trajectories, the changes in cross-sections shapes and the amplitude of shrinkage can be seen on these figures. The evolution of the tows cross-sections shape is interesting to notice : while these crosssections were initially elliptical, they take a lenticular shape in the plain weave, and various elongated shapes in the twill weave. In Fig. 14, which shows different slices of the same tow from the twill weave, the various shapes of cross-sections can be observed. This demonstrates the ability of the model to allow the fibers to rearrange in function of the loads applied to them.

The rearrangement of fibers is even allowed at the end of tows (Fig. 15) where boundary conditions are applied. This proves the effectiveness of the binding of ends of fibers to rigid bodies by means of averaged conditions : loads and displacements are globally prescribed to the barycenter of the fiber ends of the group, but they keep the possibility to move and rearrange.

\subsection{Simulation of loading cases}

Various loading cases can be simulated by applying different loads (displacements or forces) to the edges of the woven samples after simulating the weaving process. 


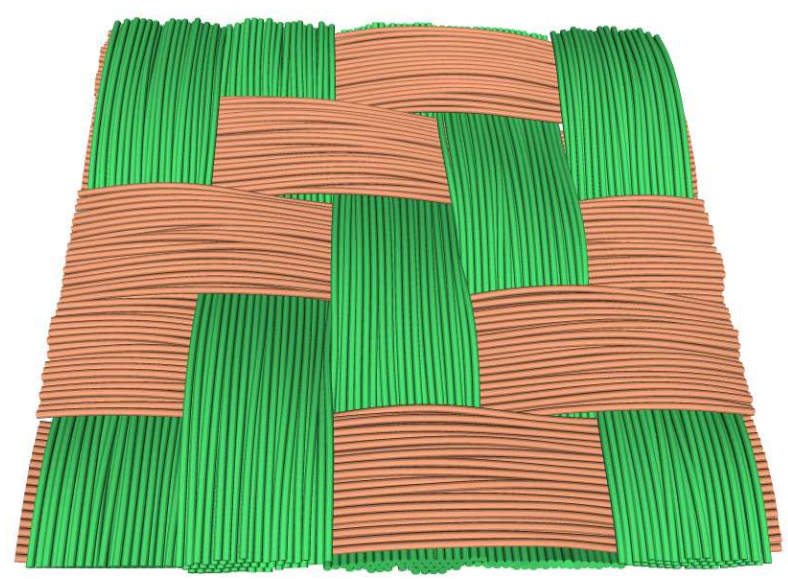

Fig. 11 Configuration after weaving for the twill weave sample
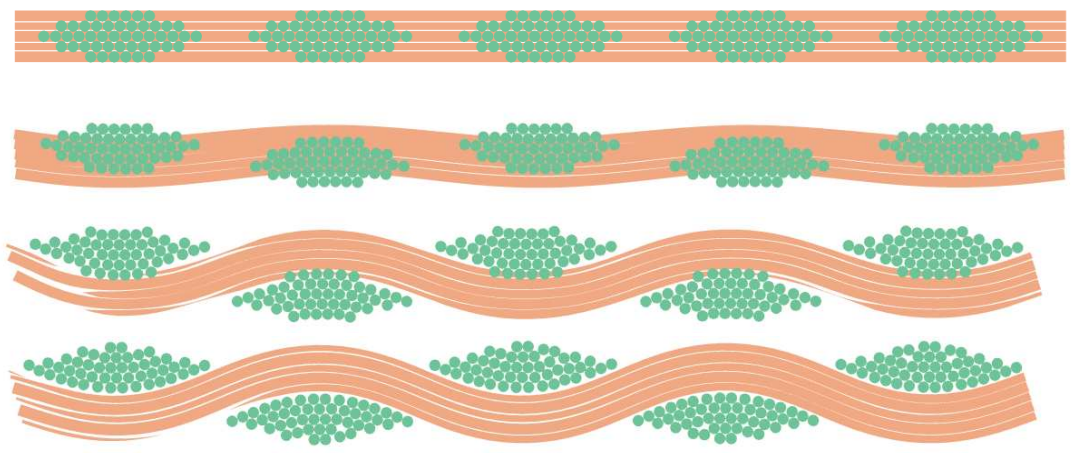

Fig. 12 Slices at successive steps during the simulation of weaving for the plain weave
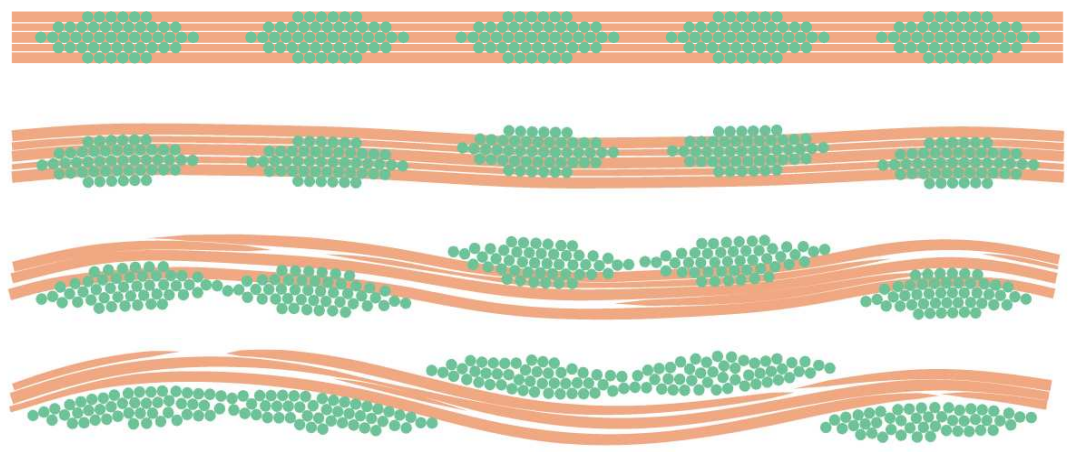

Fig. 13 Slices at successive steps during the simulation of weaving for the twill weave 

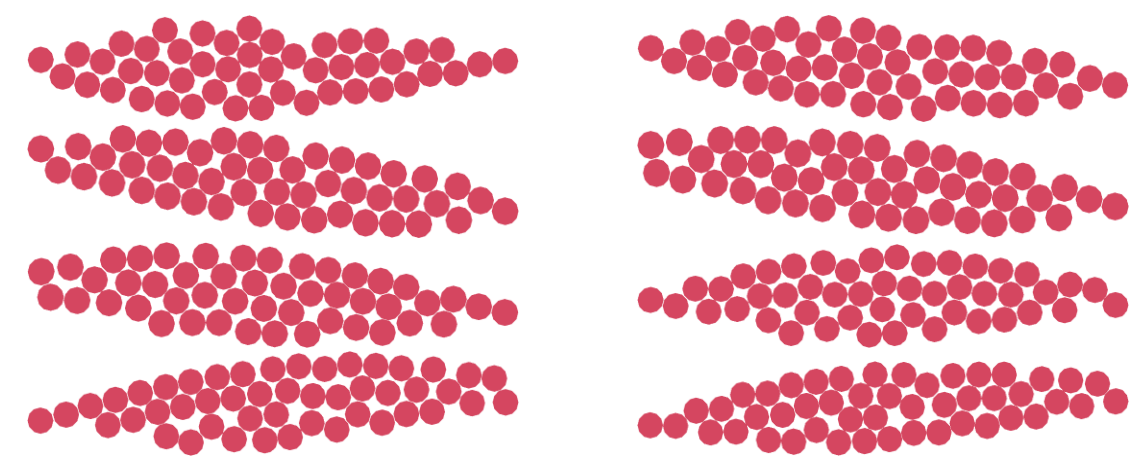

Fig. 14 Successive slices on the same tow of the twill weave
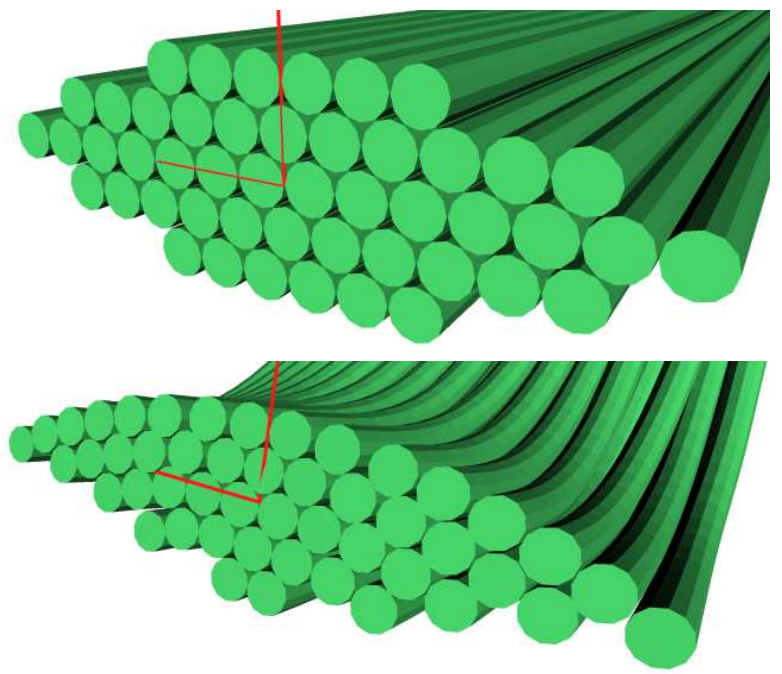

Fig. 15 Rearrangement of fibers at the end of a tow allowed by averaged boundary conditions : configurations before (top) and after (bottom) weaving

\subsubsection{Biaxial tensile tests}

Biaxial tensile tests have been simulated for the two samples of plain and twill weave. Two different ratios between the elongations applied in warp and weft directions were used : a ratio $\alpha=1$ for equibiaxial tensile tests, and a ratio $\alpha=0.5$. Loadings curves for the two ratios are plotted on Fig. 16 for the plain weave and on Fig. 17 for the twill weave. The loading curves exhibit the usual J-shape aspect at the beginning. This nonlinear effect is certainly due to the filling of free gaps between fibers as the tensile load increases, until the arrangement of fibers within tows reaches a maximum density.

\subsubsection{Shear tests}

To simulate a shear tests, opposite horizontal displacements have been applied to the top and bottom edges of the samples, while lateral edges were left free to rotate around 


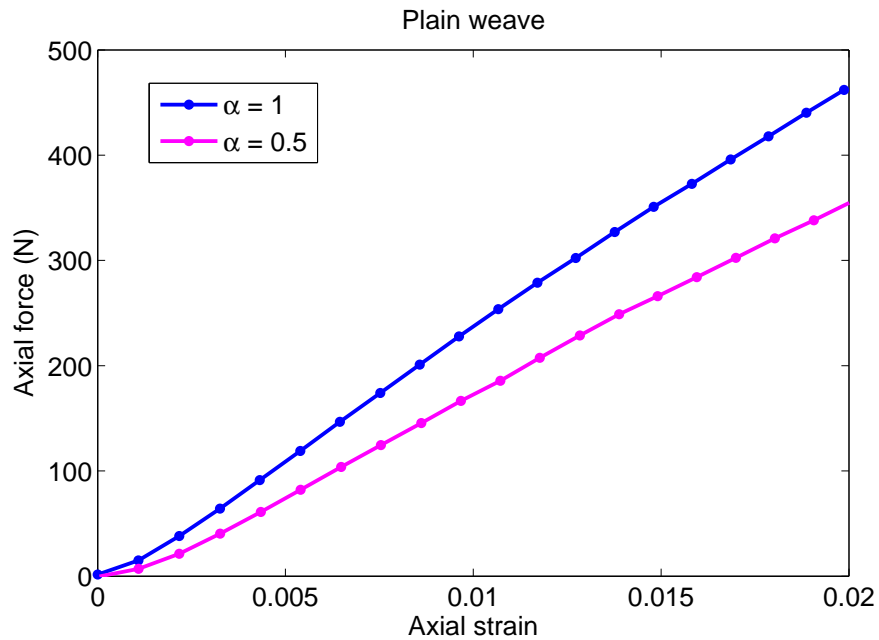

Fig. 16 Loading curves for the biaxial tensile test on the plain weave

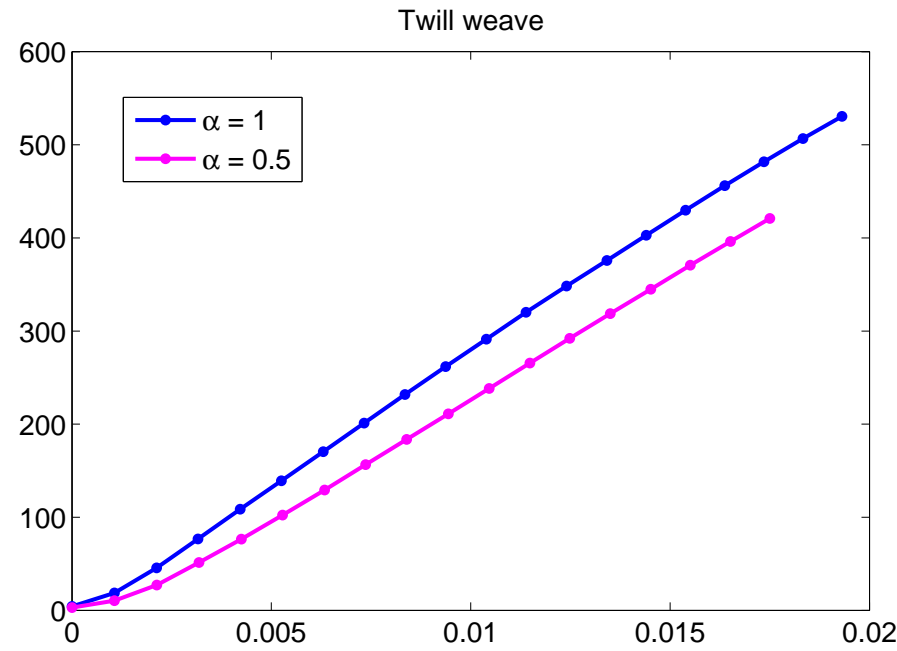

Fig. 17 Loading curves for the biaxial tensile test on the twill weave

an axis orthogonal to the plane. A shear angle of nearly $25^{\circ}$ is reached. Configurations at the end of the shear test are shown in Fig. 18 and 19. The shear loading curves (Fig. 20 ) show similar behaviours for the two samples at the begining of the loading, while the plain weave becomes stiffer as the shear angle increases. This difference between the two weave architectures may be due to the fact that the locking between tows is more severe for the plain weave than for the twill weave. 


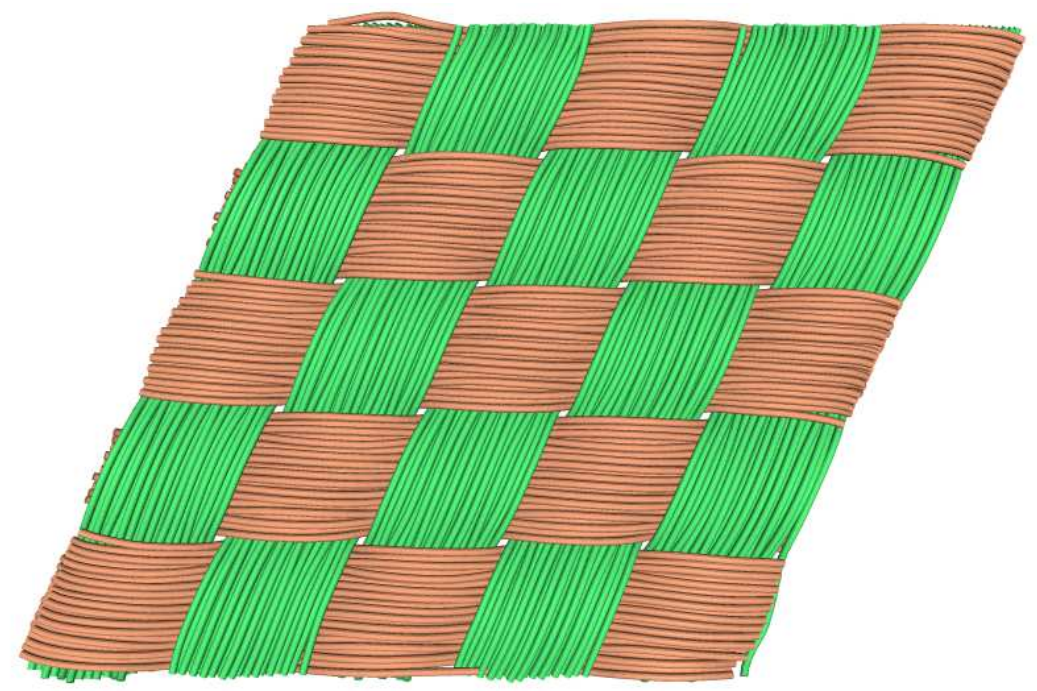

Fig. 18 Configuration at the end of the shear test for the plain weave

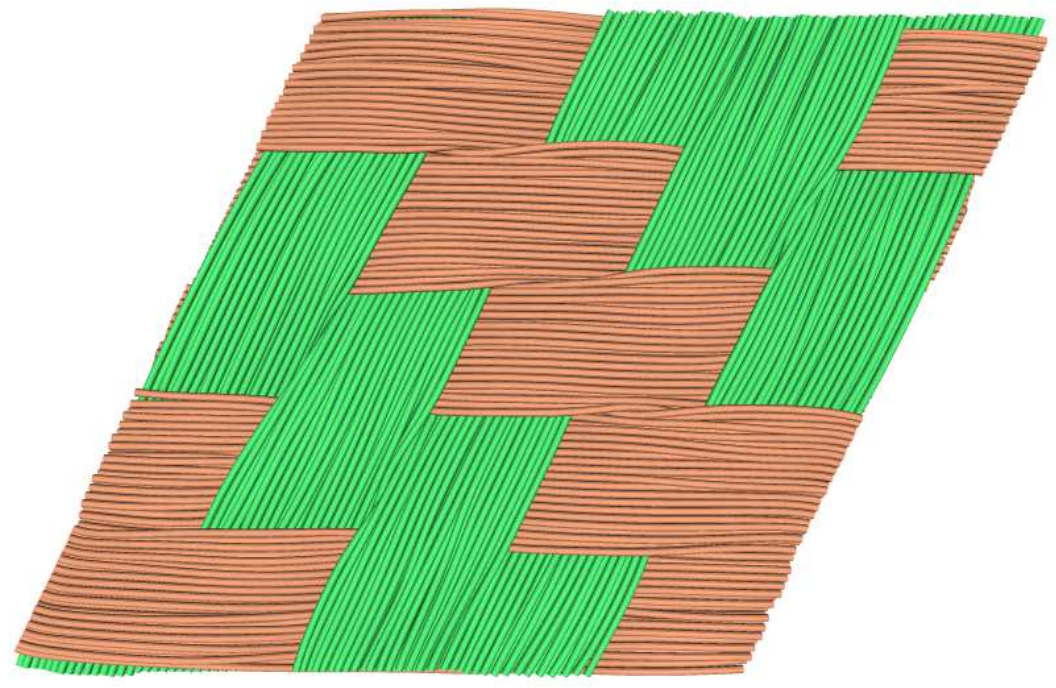

Fig. 19 Configuration at the end of the shear test for the twill weave

\section{Conclusions}

A general approach to the mechanical behaviour of woven fabrics by means of finite element simulation has been presented in this paper. This approach, formulated within a large deformations framework, is based on the representation of all fibers constituting woven structures by means of $3 \mathrm{D}$ beam models, and on the taking into account of contact-friction interactions between fibers.

The detection of the numerous contacts occurring within collections of fibers is an essential point of the approach. Based on the determination of proximity zones 


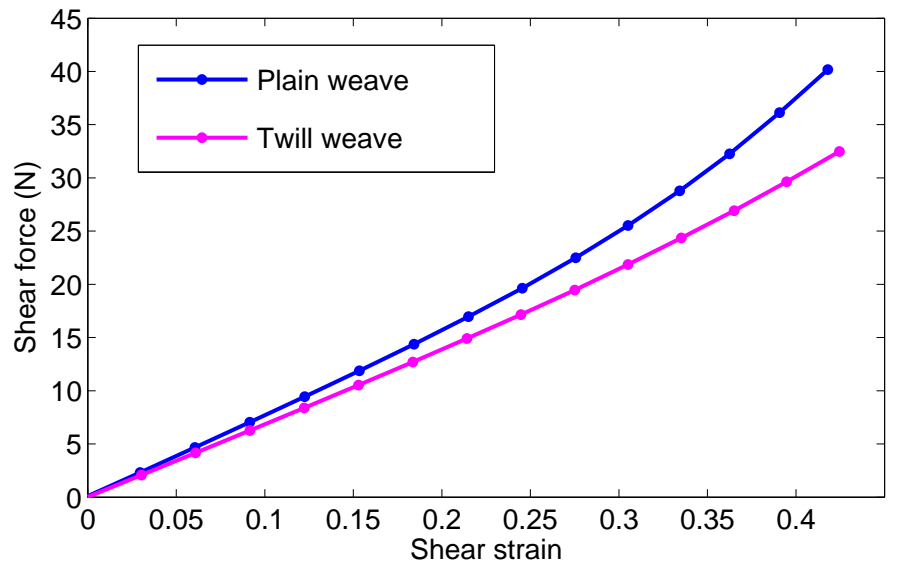

Fig. 20 Loading curves for the shear test

between fibers, and on the construction of intermediate geometries to approximate the actual contact zones, the presented method generates automatically contact elements made of pairs of material particles which are candidates to contact. The process of determination of contact elements has to be repeated as the solution evolves. It is sufficiently general to apply to very different configurations between fibers, from parallel to crossings fibers.

Robust models and efficient algorithms have been developed to ensure a good convergence for the solution of the global nonlinear problem. These optimized models and algorithms allow to consider samples of woven fabrics made of few hundreds of fibers and involving up to 100000 contact elements, with a reasonable CPU time.

The ability of the method is demonstrated first by the determination of the initial configuration of woven fabrics by simulating the weaving process. Useful informations about the trajectories and cross-section shapes of tows, and about the trajectories and curvatures of fibers, are obtained as results from this initial simulation. The simulation of various loading cases in order to identify the mechanical properties of fabrics shows that the model can consider large deformations.

The proposed modeling, based on the identification of very few parameters (mechanical properties of fibers, definition of tows arrangement and of the weave pattern), appears as a suited tool to describe and to understand complex phenomena occurring at the scale of fibers, and to predict the complex global mechanical behavior of textile materials at a macroscopic scale. This kind of simulation may be very useful to study the influence on different design parameters on the mechanical behaviour of textile fabrics.

The approach can also be applied to different mechanical problems involving assemblies of fibers. It should be helpful to study the mechanical behaviour of individual tows, or even to simulate the spinning of fibers to form yarns.

\section{References}

1. B. Ben Boubaker, B.H., Ganghoffer, J.F.: Mesoscopic fabric models using a discrete massspring approach: Yarn-yarn interactions analysis. Journal of Materials Science 40(22), 
$5925-5932(2005)$

2. Badel, P., Vidal-Salle, E., Boisse, P.: Computational determination of in-plane shear mechanical behaviour of textile composite reinforcements. Computational Materials Science 40(4), 439-448 (2007)

3. Ballhause, D., König, M., Kröplin, B.: A microstructure model for fabric-reinforced membranes based on discrete element modelling. In: E.O. nate, B. Kröplin (eds.) Textile Composites and Inflatable Structures II, pp. 255-263. Structural Membranes 2005, CCIMNE, Barcelona, Stuttgart, Germany (2005)

4. Boisse, P., Zouari, B., Gasser, A.: A mesoscopic approach for the simulation of woven fibre composite forming. Composites Science and Technology 65(3-4), 429-436 (2005)

5. Bridgens, N., Gosling, P., Birchall, M.: Membrane material behaviour: concepts, practice and develoments. The Structural Engineer 82(14), 28-33 (2004)

6. Durville, D.: Numerical simulation of entangled materials mechanical properties. Journal of Materials Science 40(22), 5941-5948 (2005)

7. Durville, D.: Recent Advances in Textile Membranes and Inflatable Structures, chap. Finite element simulation of the mechanical behaviour of textile composites at the mesoscopic scale of individual fibers, pp. 15-34. Springer (2008)

8. Finckh, H.: Numerical simulation of mechanical properties of fabrics - weaving / numerische simulation der mechanischen eigenschaften textiler flächengebilde - gewebeherstellung. Proceedings of the German 3rd LS-DYNA Forum 2004, Bamberg, Germany (2004)

9. Miao, Y., Zhou, E., Wang, Y., Cheeseman, B.A.: Mechanics of textile composites: Microgeometry. Composites Science and Technology 68(7-8), 1671-1678 (2008)

10. Wriggers, P., Zavarise, G.: On contact between three-dimensional beams undergoing large deflections. Communications in Numerical Methods in Engineering 13, 429-438 (1997)

11. Zavarise, G., Wriggers, P.: Contact with friction between beams in 3-d space. International Journal for Numerical Methods in Engineering 49, 977-1006 (2000) 